\title{
sciendo
}

\section{MULTISCALE FOURIER LANDSCAPE PATTERN INDICES FOR LANDSCAPE ECOLOGY}

\author{
ALVIN SPIVEY, ANTHONY VODACEK \\ Rochester Institute of Technology, 54 Lomb Memorial Drive, Rochester, NY 14623, e-mail: \\ alvin.spivey@gmail.com
}

Received: $16^{\text {th }}$ December 2016, Accepted: $31^{\text {th }}$ July 2018

\begin{abstract}
A factor analysis of 67 landscape pattern metrics was performed to quantify the ability of landscape pattern metrics to explain land cover pattern, and to report individual landscape pattern metric values that are statistically independent. This land cover pattern is measured from $7.68 \times 7.68[\mathrm{~km}]$ GeoTiff image tiles of the conterminous United States Geological Survey (USGS) 1992 National Land Cover Dataset (NCLD). Using factor analysis to rank independent landscape pattern information, each landscape pattern metric produces the explanatory power of that landscape pattern metric amongst the other 66 landscape pattern metrics - any landscape pattern metrics that report similar values contribute redundant information. The metrics that contribute the most information are Jackson's Contagion statistic (P005), typically contributing to $97 \%$ of the explained variability; the Fourier Metric of Fragmentation ( $F M F)$, typically contributing to $65 \%$ of the explained variability; and average $L C L U$ class lacunarity (TLAC), typically contributing to $62 \%$ of the explained variability. Two other Fourier-based landscape pattern metrics we tested, the Least Squares Fourier Transform Fractal Dimension Estimation ( $L S F T$ ) and the Fourier Metric of Proportion $(F M P)$, contributed $50 \%$ and $12 \%$ to the explained variability, respectively. In addition, the values reported by each of the Fourier metrics are revealed to be relatively independent amongst commonly used landscape pattern metrics and are thus demonstrated to be appropriate for explaining general landscape pattern variability.
\end{abstract}

Keywords: Fourier, Landscape Pattern Metric, Factor Analysis, NLCD.

\section{INTRODUCTION}

Interpreting the effects of landscape pattern on ecosystem function is not a new adventure in ecological study. A number of researchers have successfully related landscape pattern metrics to environmental variables. Landscape pattern has been broadly interpreted to relate to the status of a variety of environmental variables - including identifying the presence of nitrogen, phosphorus, and lead, and measuring chlorophyll nutrient loads, fish diversity, insect density, and broader invertebrate density (Detenbeck et al., 1993; Johnes et al., 1996; John-son et al., 1997; Klein, 1979; Osborne \& Wiley, 1988; Schueler \& Galli, 1992; Sponseller et al., 2001; Wang et al., 1998; Woodwell, 1998). Each of these environmental variables have been correlated to landscape pattern to within $65 \%$ to $85 \%$ of their total variability (Jones et al., 2001). 
Examples of the landscape pattern analysis approach can be found for environments as diverse as the watersheds of Brazil's Amazon and urban streambeds in Maryland, USA. In two studies by Williams et al. (1997) and Williams \& Melack (1997) an inverse correlation between increases in Rodoñia, Brazil presence of forest land cover landscape pattern metric and stream water nitrogen-to-phosphorus nutrient ratios were observed for peak stream flow storm events-making the presence of forest land cover landscape pattern metric a strong indicator of nutrient flow. In a similar study, but in a developed area of Maryland, USA during drought and wet years, nitrate exports to streams showed significant variation in streams depending on whether the adjacent land use was suburban or urban (Kaushal et al., 2010; 2008), allowing the authors to predict stream hypoxic zones using an along stream land use landscape pattern metric. The basic approach taken in these experiments followed three stages:

1. Identification of appropriate landscape pattern metrics,

2. Identification of indicator variable(s) and selecting the appropriate landscape pattern metric that best describe the variable(s), and

3. Interpreting the impacts of the indicator variables on environmental status or processes.

The landscape pattern metrics used in these experiments were interpreted as important measures of landscape response to environmental pressures, thus tying the landscape pattern to the ecological function. Choosing appropriate landscape pattern metrics is a critical first stage in these experiments, but the decision on which metric(s) will be appropriate among the many published landscape pattern metrics (Neel et al., 2004; Wu, 2004; Wu et al., 2002) was made for location and scale specific applications, with the aide of expert knowledge. Further, a metric may be shown to be a reliable description of landscape pattern, but when multiple metrics are examined, no additional information is gained when a metric is similar or exactly the same as another (see Spivey (2011) for further landscape pattern reliability discussion). To address these types of problems, Ritters et al. (1995) designed a factor analysis where they statistically compared multiple landscape pattern metrics to find how well each metric responded to relevant land cover map change.

In this paper, we further explore the identification of appropriate landscape pattern metrics for broad scale and environmental applications, and we describe three new landscape pattern metrics based on Fourier analysis. First, we introduce the formulation and rationale for our three Fourier landscape pattern metrics. Second, we use a correlation analysis to assess the extent to which independent information is provided by a suite of 67 landscape pattern metrics, compounded from previously published landscape pattern metrics, plus our three new metrics. Third, we use the factor analysis to test which of the landscape pattern metrics have the best explanatory value for land cover variability in the National Land Cover Database (NLCD). The NLCD informs our metrics with broad variability within the 48 conterminous Unied States ecoregions. Ecoregions classified into 14 different biomes - such as forests, grassland, or deserts.

Our analysis thus extends the work of Ritters et al. (1995) by testing for both independence as well as explanatory power among the metrics over a wide range of land cover types. Location and scale specific trade studies are useful for certain applications of landscape pattern, whose results may not be able to be generalized. Here, we have identified Landscape Pattern Metrics that are statistically useful for general use over a wide variety of land cover types, and thus useful for understanding broad scale processes like climate change, desertification, and urban sprawl. 


\section{METHODS}

\section{National Land Cover Data}

This work requires an extensive and diverse spatial dataset on which to apply landscape pattern metrics. We use the 1992 United States Geological Survey National Land Cover Data (NLCD), which is a 21-category land cover classification scheme that was applied across a set of Landsat satellite images of the 48 conterminous United States (Vogelmann et al., 2001). The NLCD 1992 classification data are provided as 256 × 256 pixel GeoTIFF image tiles (see Fig. 1 for an example image tile). At the Landsat spatial resolution of $30 \mathrm{~m}$ each tile represents $7.68 \times 7.68[\mathrm{~km}]$ areas and there are more than 160,000 separate tiles covering a very diverse set of 85 ecoregions. These ecoregions are shown in Fig. 2.

Fig. 1: A 256 x 256 tile of the National Land Cover Database (NLCD) in Glens Falls, New York, USA. The Anderson et al. (1976) Level II attribute class description and the land cover class color code are shown along the right side.

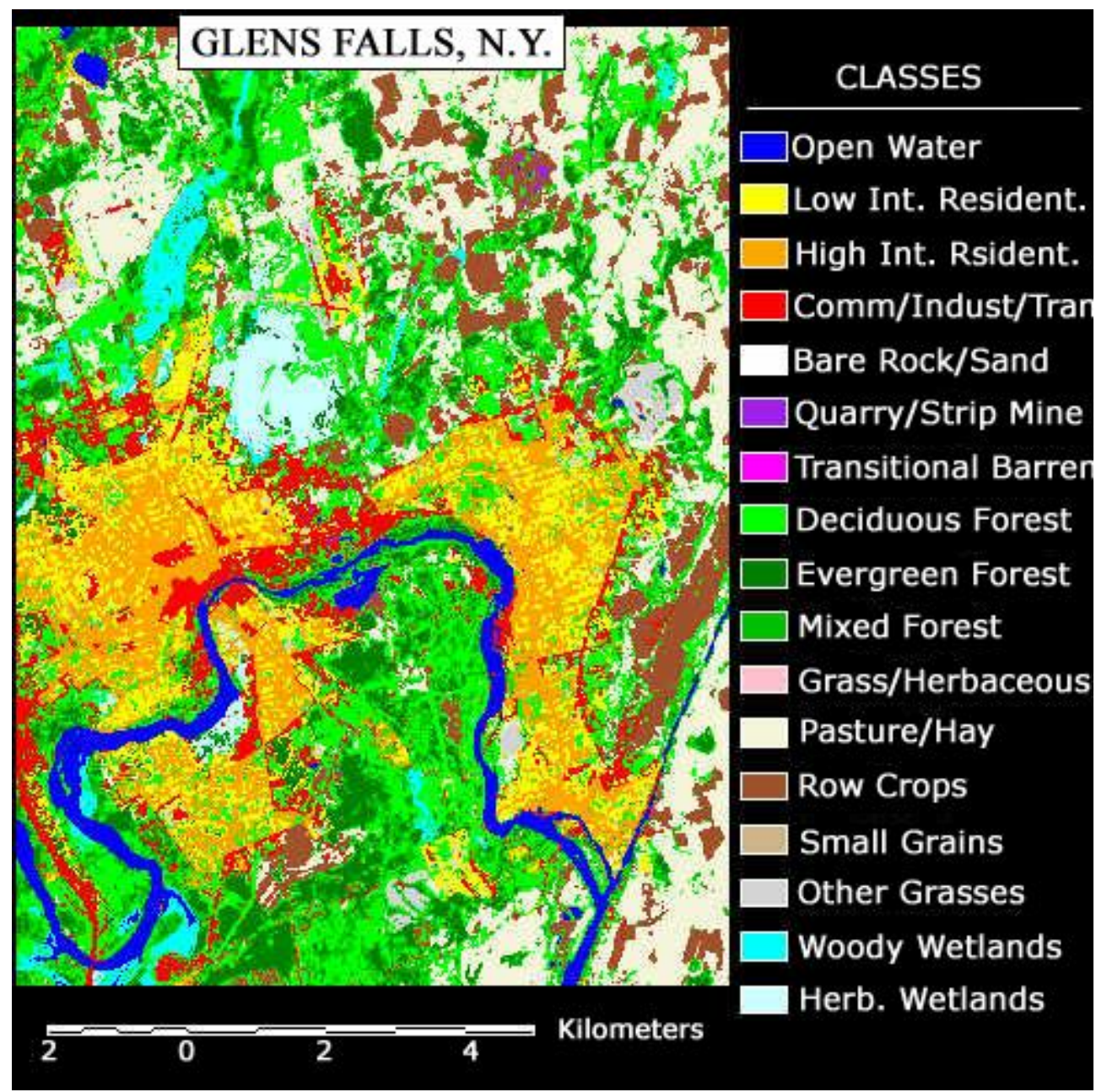


Fig. 2: Ecoregions. The 48 conterminous United States consists of 85 of the 867 global terrestrial ecoregions. The ecoregions are classified into 14 different biomes-such as forests, grassland, or deserts - making ecoregions descriptive of land cover data.

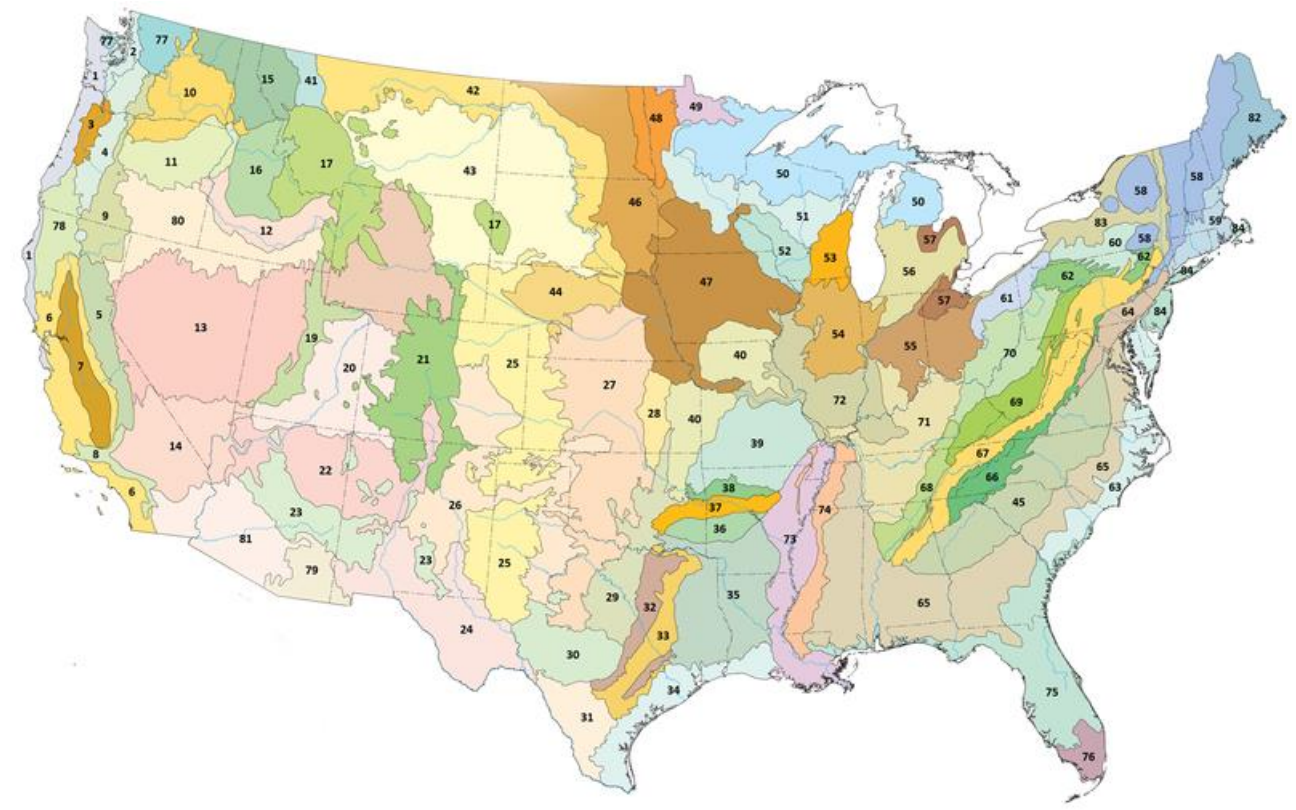

\section{Landscape Metrics}

Every one of the 67 landscape pattern metrics were applied to each $7.68 \times 7.68[\mathrm{~km}]$ NLCD tile (more than 160,000 tiles). A reference to each landscape pattern metric obtained from the literature, as well as the details of each formulation can be found in Appendix A. A summary of our three new multiscale Fourier landscape pattern metrics is presented below. More details on each of these metrics can be found in Spivey (2011).

\section{Fourier Metric of Proportion (FMP)}

Derived to be an unbiased metric of scale, pixels from the observed land cover class $j$ are divided by the total number of land cover pixels being observed:

$$
\begin{aligned}
F M P_{j} & \equiv \prod_{j}^{D} p_{j}^{\prime} \\
& =\prod_{j}^{D} \frac{\text { total number of pixels in class } j}{\text { total number of pixels in largest class }}
\end{aligned}
$$

In a landscape composed of land cover patches, $D$ would be the total number of patches observed, and $p_{j}{ }_{j}$ the ratio between the number of pixels in the class of interest $j$ and the number of pixels in the largest class observed. As a measure of landscape composition, this type of denominator references land cover class $j$ pixel counts to the largest, most scale-resistant land cover class being observed.

\section{Fourier Metric of Fragmentation (FMF)}

Designed according to the concept of landscape contagion, the Fourier metric of fragmentation measures clumping and interspersion between two classes. The ecological interpretation of contagion is that it indicates the dynamics between two land cover classes. 
This landscape configuration metric is reported as the distance between two land cover class maps by name (see Spivey \& Vodacek (2017) for an example). The contagion metric measures patch adjacencies, making it impossible to measure clumping and interspersion between distant classes. This is not a problem for $F M F$. These land cover classes are explicit in the equation below:

$$
\begin{aligned}
& F M F_{l, j, k} \equiv M_{j, k}\left(1-\Phi_{j, k}^{l}\right) \\
& =\frac{\text { total number of pixels in class } j}{\text { total number of pixels in class } j, \text { and } k} \times \\
& \left(1-e^{\left[\ln \left(\mathcal{F}\left\{L C_{j}\right\} \div \mathcal{F}\left\{L C_{k}\right\}\right)-\ln \left(\sqrt{M^{2}+N^{2}}\right)\right]^{l}}\right)
\end{aligned}
$$

Here, each land cover $L C_{j}$ and $L C_{k}$ are binary class maps of $0^{\prime} s$ and $1^{\prime} s$, the Fourier operator is shown as $\mathcal{F}\{\cdot\}$, and the parameters $M$ and $N$ are the image tile number of row pixels and column pixels respectively. The equation variables include, $M_{j, k}$ a ratio between the total number of pixels in the class of interest and total number of pixels between the two classes being compared; and $\Phi_{j, k}$ a variable that includes the LOG Fourier phase difference between individual class maps $L C_{j}$ and $L C_{k}$, all log-normalized by the largest distance possible in the observed class map (i.e. $\sqrt{M^{2}+N^{2}}$ ). The metric parameter $l$ increases or decreases the migration rates being modeled by the metric, see Spivey \& Vodacek (2017) for details.

\section{Yuen et al. (2004) Least Squares Fourier Transform Fractal Dimension Estimation}

For fractal dimension estimation of statistically self-similar land cover classification patches, we adopt the method of Yuen et al. (2004) for analyzing the Fourier power spectrum of a 1-D signal to our case of a 2-D land cover image. This landscape pattern metric measures patch structure. After linearizing the Fourier power spectrum, the Hough transform is used to estimate the Hurst dimension $H$ and thereby fractal dimension $D_{f r}$.

The 2-D Fourier power spectrum $P_{f r}[\xi, \eta]$ for a Fourier fractal signal $F_{f r}[\xi, \eta]$ is statistically equal to the least squares Fourier power spectrum estimator:

$$
\begin{aligned}
P_{f r}[\xi, \eta] & =\left|F_{f r}[\xi, \eta]\right| 2 \\
& \equiv \frac{A}{\left(\xi^{2}+\eta^{2}\right)^{H+1}}, \text { where } D_{f r}=3-H
\end{aligned}
$$

$A$ is a proportional constant, $D_{f r}$ is the fractal dimension, and $H$ is the Hurst exponent.

The true fractal signal $f_{f r}[x, y]$ and fractal noise $\epsilon[x, y]$, have power spectrum $P_{f r}[\xi, \eta]$ and $\epsilon[\xi, \eta]$, respectively. The composite signal $f[x, y]=f_{f r}[x, y]+\epsilon[x, y]$ has power spectrum $P[\xi, \eta]$ :

$$
P[\xi, \eta]=P_{f r}[\xi, \eta]+\epsilon[\xi, \eta]
$$

Solving for $P_{f r}[\xi, \eta]$, and taking the logarithm of both sides, linearizes the least squares Fourier power spectrum equation:

$$
\begin{aligned}
& \log [P[\xi, \eta]] \approx \log [A]-(H+1) \cdot \log \left[\xi^{2}+\eta^{2}\right] \\
& \text { So, } \\
& \log [A]=(H+1) \cdot \log \left[\xi^{2}+\eta^{2}\right]+\log [P[\xi, \eta]]
\end{aligned}
$$


In the last step, fractal noise $\epsilon[x, y]$ is assumed negligible. The least squares Fourier transform method for estimating fractal dimension takes advantage of the Hough transform's precision. Each possible linear trend is counted in Hough space, then the most likely linear trend is accepted for derivation of the Hurst parameter $H$ and thereby the fractal dimension $D_{f r}=3-H$.

\section{Factor Analysis}

The factor analysis was done in SAS and describes the covariance relationships among many landscape pattern metrics in terms of observable, random land cover variability. Since a map of land cover is analyzed to create the landscape pattern metric, land cover variability is captured in the metric values. The factor analysis uses Principal Component Analysis (PCA) to determine these covariance relationships. For a more complete handling of PCA refer to Richards \& Jia (1996).

Each image tile produces 67 separate measurements. Using this data, a vector space can be created with as many dimensions as there are image tiles, associated with their 67 landscape pattern measurements. For an individual tile, the $p=67$ landscape pattern metrics produce a column vector:

$$
\mathbf{x}=\left[\begin{array}{c}
x_{1} \\
x_{2} \\
\vdots \\
x_{p}
\end{array}\right]
$$

By calculating the mean of each landscape pattern metric over the full dataset of land cover tiles a mean vector with $p=67$ elements and a square covariance matrix are formed:

$$
\begin{gathered}
\boldsymbol{\mu}=\left[\begin{array}{c}
\mu_{1} \\
\mu_{2} \\
\vdots \\
\mu_{p}
\end{array}\right] \\
\boldsymbol{\Sigma}=\left[\begin{array}{ccccc}
\sigma_{1,1} & \sigma_{1,2} & \sigma_{1,3} & \ldots & \sigma_{1, p} \\
\sigma_{2,1} & \sigma_{2,2} & \sigma_{2,3} & \ldots & \sigma_{2, p} \\
\sigma_{3,1} & \sigma_{3,2} & \sigma_{3,3} & \ldots & \sigma_{3, p} \\
\vdots & \vdots & \vdots & \ddots & \vdots \\
\sigma_{p, 2} & \sigma_{p, 2} & \sigma_{p, 2} & \ldots & \sigma_{p, p}
\end{array}\right]
\end{gathered}
$$

The factors that cause these landscape pattern metrics to vary can be multiple, including the most obvious land cover variability, there could also be influences from large weather events or local public policy. According to the factor analysis, there can be $M<p$ Factors that effect the reported landscape pattern metrics. This is best modeled also as a column vector, seen in Equation 9.

$$
\mathbf{F}=\left[\begin{array}{c}
F_{1} \\
F_{2} \\
\vdots \\
F_{M}
\end{array}\right] .
$$

The degree of effect for each factor on individual landscape pattern metrics can be estimated. This is done using the spectral model shown in Equation 10. 


$$
\begin{aligned}
x_{1} & =\mu_{1}+\lambda_{1,1} F_{1}+\lambda 1,2 F_{2}+\cdots+\lambda_{1, M} F_{M}+\epsilon_{1} \\
x_{1} & =\mu_{1}+\lambda_{1,1} F_{1}+\lambda 1,2 F_{2}+\cdots+\lambda_{1, M} F_{M}+\epsilon_{2} \\
\vdots & \vdots \\
x_{p} & =\mu_{p}+\lambda_{p, 1} F_{1}+\lambda p, 2 F_{2}+\cdots+\lambda_{p, M} F_{M}+\epsilon_{p}
\end{aligned}
$$

Equivalently, in matrix form, we have the factor analysis factor model:

$$
\begin{array}{rrr}
\mathbf{x} & =\boldsymbol{\mu}+\boldsymbol{\Lambda F}+\boldsymbol{\epsilon} \\
\mathbf{x}-\boldsymbol{\mu} & = & \boldsymbol{\Lambda F}+\boldsymbol{\epsilon}
\end{array}
$$

This de-meaned data $\mathbf{x}-\boldsymbol{\mu}$, is what is used as input into SAS FACTOR analysis statistical software. The output is a square matrix of explained variance.

Each parameter along the diagonal of the $\boldsymbol{\Psi}$ square matrix, is the explained variance for the landscape pattern metric of that row. Using each diagonal term, a measure of explained variance can be obtained. Equation 12 shows how this estimation of landscape pattern metric fidelity would work, using the $\sigma_{j, j}$ elements of the landscape pattern metric covariance matrix in Equation 8 above.

$$
\boldsymbol{\Psi}=\left[\begin{array}{ccccc}
\Psi_{1,1} & 0 & 0 & \ldots & 0 \\
0 & \Psi_{2,2} & 0 & \ldots & 0 \\
0 & 0 & \Psi_{3,3} & \ldots & 0 \\
\vdots & \vdots & \vdots & \ddots & \vdots \\
0 & 0 & 0 & \ldots & \Psi_{p, p}
\end{array}\right]
$$

This metric $\bar{\sigma}$ tells us how much each factor $\Psi$ explains the variance $\sigma$ of the landscape, for the metric $j$. Because this metric of Expected Correlation Contribution (ECC) describes the covariance relationships $\sigma_{j, j}$ among many landscape pattern, and many of the metrics are not statistically independent, the sum of the fidelity metric $\bar{\sigma}_{j}$ does not sum up to $100 \%$. That is to say, some metrics vary in the same way as other metrics - those metrics would share the same $\bar{\sigma}_{j}$ value. This issue of statistical independence for interpretability is discussed in the next section.

\section{Independence of Fourier Landscape Pattern Metrics}

Another way the data were analyzed was to look at the relationship between the 160,000 tiles. A count of how often each landscape pattern metric was correlated to another is shown for increasing degrees of similarity in Figure 3, Figure 4, and Figure 5. The most independent metrics are those with near 0 correlation counts. The most independent of these metrics are underlined in Table 2. 
Fig. 3: The number of landscape pattern metrics correlated greater than $\mathbf{7 0}$ points. Fourier metrics $L s F T, F M P$, and $F M F$ are correlated to 46, 5, and 12 other landscape pattern metrics, respectively.

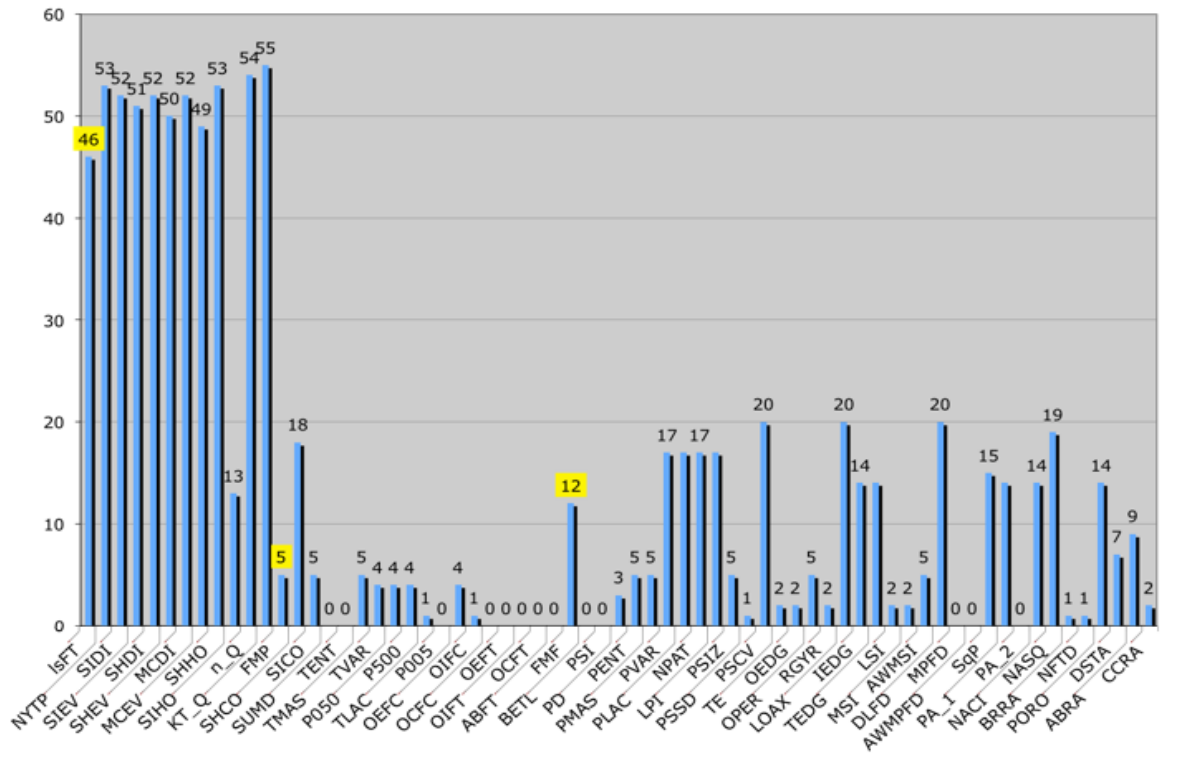

Fig. 4: The number of landscape pattern metrics correlated greater than 85 points. Fourier metrics LsFT, FMP, and FMF are correlated to 29, 5, and 12 other landscape pattern metrics, respectively.

$>.85$ Correlation

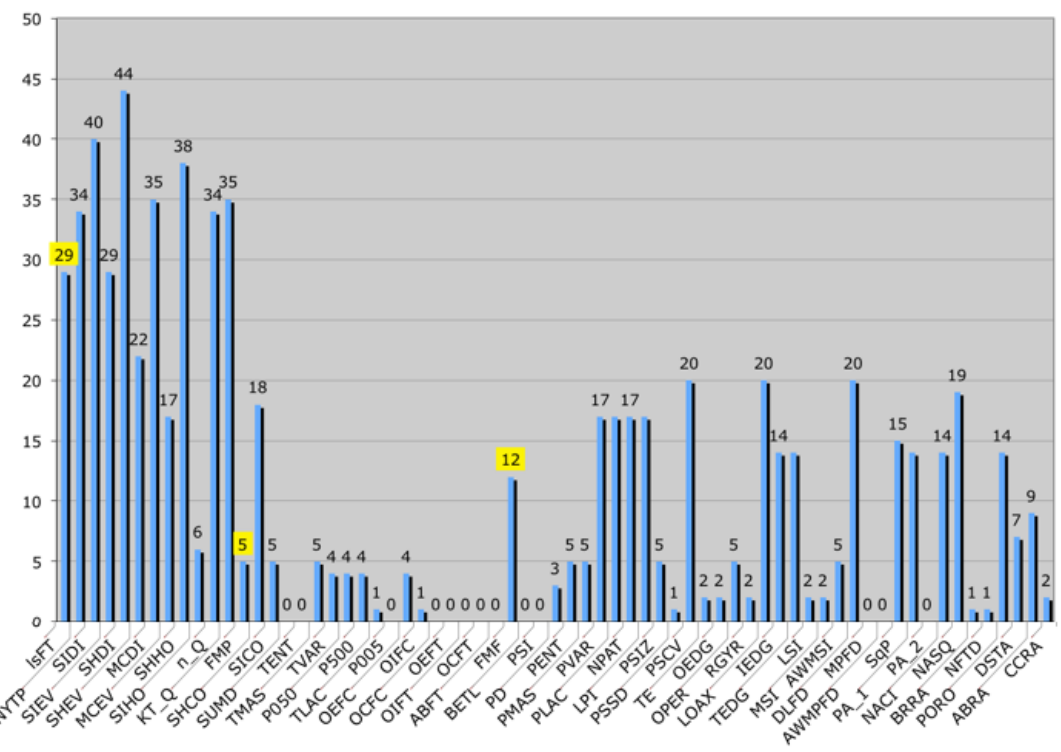


Fig. 5: The number of landscape pattern metrics correlated greater than 99 points. For Fourier metrics LsFT, FMP, and FMF are correlated to 0, 5, and 12 other landscape pattern metrics, respectively.
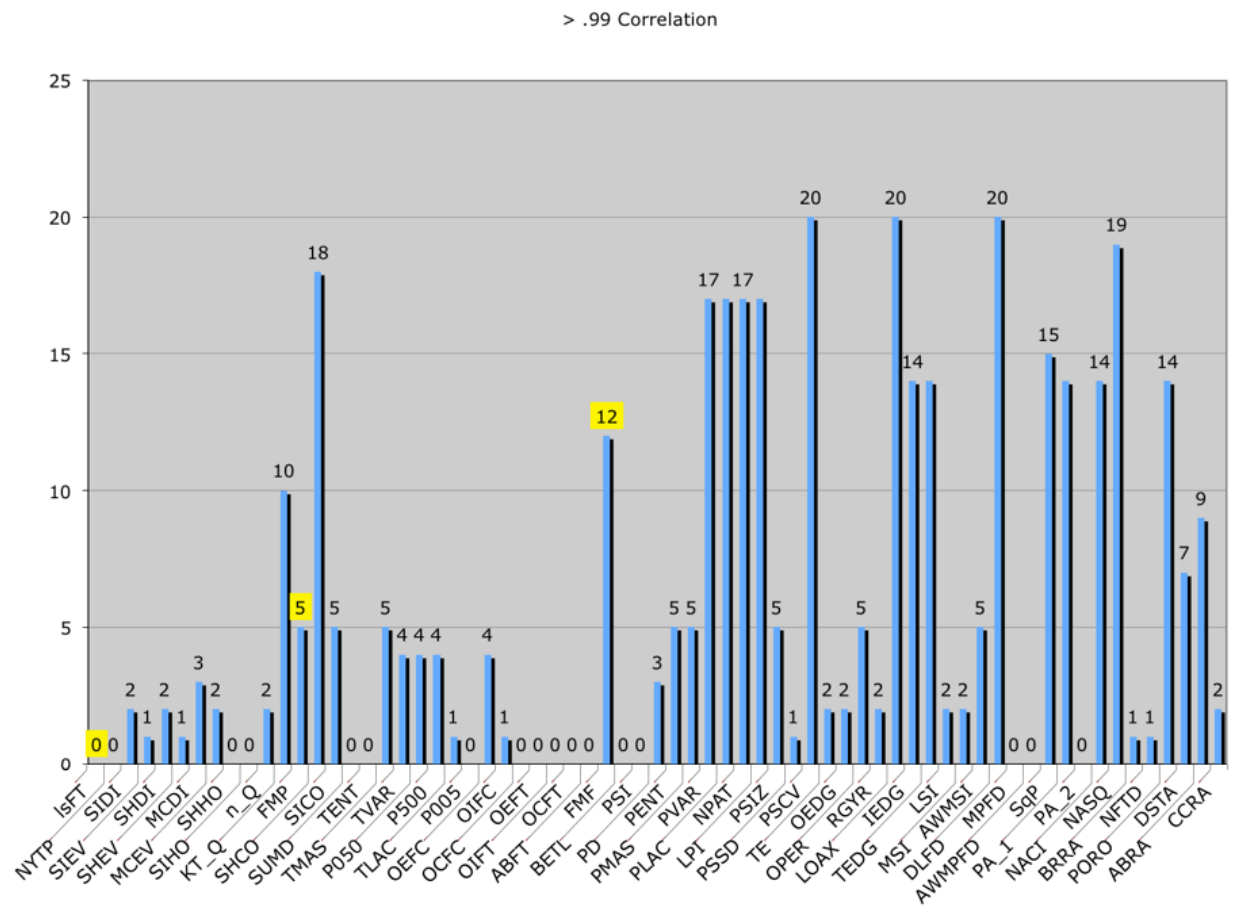

The least frequently correlated Fourier metric is LsFT. This small amount of between metric correlation is not observed in every Fractal Dimension landscape pattern, like those seen in Appendix A.5, A.8, and A.9 of Spivey (2011). Thus LsFT is the most independent Fourier description of landscape fractal dimension. The most correlated Fourier metric is $F M F$. These $F M F$ results were the least independent Fourier description of landscape pattern. The four landscape pattern metrics most correlated to the Fourier metrics are shown in Table 1.

The four leading metrics in Table 1 seem to report redundant Fourier landscape pattern information, though none of them have as physical a description of pattern and processes as their Fourier relatives. For instance, though FMP values are very related to $S H C O, n-Q$, $P S C V$, and $L O A X$ only FMP is directly related to and interprets itself along the physical migration and extinction rates between land cover patches. Meanwhile, the Contagion $\mathrm{SHCO}$ method has shown itself to be highly dependent on image sensor error and so is unreliable. $n-Q$ is an inter-quartile abundance that has only a mathematical, no physical interpretation. And, both PSCV and LOAX measure of how different patch sizes are within a landscape, using the unreliable nearest-neighbor patch definition (see Sections 3.2.4 and 5.2 of Spivey (2011) for a discussion on the errors in this nearest-neighbor approach, and on how unreliable Contagion $\mathrm{SHCO}$ performance can be). 
Table 1: The top four landscape pattern metrics most correlated to the Fourier metrics when observing seamless conterminous United States NLCD 1992 LCLU

\begin{tabular}{ccc}
\hline \hline LsFT & FMP & FMF \\
\hline PORO & SHCO & DLFD \\
TEDG & n-Q & NASQ \\
IEDG & PSCV & LOAX \\
KT-Q & LOAX & PSCV \\
\hline
\end{tabular}

Table 2: The landscape pattern metrics (LPM) ranked by expected contribution to the explanation of seamless conterminous United States 1992 NLCD. This ranking reveals how well each metric generally relates to landscape variability, ignoring any ability to reliably or relevantly interpret that variability. Highly independent metrics are underlined, and the Fourier metrics are in bold.

\begin{tabular}{cccc}
\hline \hline LPM & E.C.C. & LPM & E.C.C. $^{\mathrm{a}}$ \\
\hline P005 & $97.46 \%$ & & \\
FMF & $64.76 \%$ & $\underline{\text { SIHO }}$ & $7.75 \%$ \\
TLAC & $61.54 \%$ & PD & $7.39 \%$ \\
LsFT & $50.06 \%$ & $\underline{\text { OCFC }}$ & $7.01 \%$ \\
ABRA & $38.76 \%$ & RGYR & $6.52 \%$ \\
BETL & $33.04 \%$ & SICO & $6.45 \%$ \\
OIFC & $32.05 \%$ & n-Q & $6.28 \%$ \\
SqP & $30.93 \%$ & PA-1 & $6.19 \%$ \\
PVAR & $28.35 \%$ & TVAR & $6.09 \%$ \\
MPFD & $27.32 \%$ & NYTP & $6.06 \%$ \\
PSSD & $26.52 \%$ & DLFD & $5.96 \%$ \\
OIFT & $25.11 \%$ & AWMSI & $5.89 \%$ \\
PLAC & $24.80 \%$ & BRRA & $5.88 \%$ \\
OEFT & $18.72 \%$ & NFTD & $5.88 \%$ \\
OPER & $18.59 \%$ & KT-Q & $5.70 \%$ \\
NASQ & $18.35 \%$ & MCDI & $5.67 \%$ \\
PENT & $17.72 \%$ & OEDG & $5.06 \%$ \\
PMAS & $17.72 \%$ & AWMPFD & $4.83 \%$ \\
PSIZ & $17.72 \%$ & CCRA & $4.80 \%$ \\
SHDI & $15.98 \%$ & $\underline{\text { PA-2 }}$ & $4.34 \%$ \\
SHHO & $15.46 \%$ & IEDG & $4.22 \%$ \\
TMAS & $15.42 \%$ & TEDG & $4.22 \%$ \\
SUMD & $13.55 \%$ & PORO & $4.22 \%$ \\
ABFT & $13.15 \%$ & TENT & $2.84 \%$ \\
NACI & $11.94 \%$ & PSCV & $2.65 \%$ \\
FMP & $11.81 \%$ & LOAX & $2.54 \%$ \\
P050 & $11.67 \%$ & P500 & $2.14 \%$ \\
LSI & $10.48 \%$ & OEFC & $2.14 \%$ \\
DSTA & $10.42 \%$ & SHCO & $2.07 \%$ \\
SIDI & $9.67 \%$ & SHEV & $2.03 \%$ \\
MSI & $9.31 \%$ & OCFT & $1.11 \%$ \\
SIEV & $9.07 \%$ & TE & $.95 \%$ \\
PSI & $8.82 \%$ & LPI & $.61 \%$ \\
MCEV & $7.79 \%$ & NPAT & $.23 \%$ \\
\hline
\end{tabular}

${ }^{\mathrm{a}} \mathrm{ECC}$ is the Expected Correlation Contribution, as defined in equation 12 above 


\section{Explanatory Power of Landscape Pattern Metrics}

To see how well each landscape pattern metric performed using their individual definitions for relevant pattern variability we can observe the amount of explained variability each metric contributes. The ranking for all Factors is shown in Table 2. The metrics that contributed most to explained variability were Jackson's Contagion statistic (P005) typically contributing to $97 \%$ of the explained variability, the Fourier Metric of Fragmentation $(F M F)$ typically contributing to $65 \%$ of the explained variability, the average land cover class lacunarity (TLAC) typically contributing to $62 \%$ of the explained variability, and the Least Squares Fourier Transform Fractal Dimension Estimation (LSFT) typically contributing to $50 \%$ of the explained variability - the Fourier Metric of Proportion (FMP) typically contributed to about $12 \%$ of the explained variability. While landscape pattern metrics like $P 005$, and TLAC describe landscape pattern variability well, they both fall short of the criteria to measure physical variables, with P005 also using the image sensor error limited nearest-neighbor method (Spivey, 2011).

ECC is the Expected Correlation Contribution, as defined in equation 12 above.

\section{Summary of Uniqueness of Fourier Landscape Pattern Metrics}

The values reported by each of the proposed Fourier metrics have been shown to be independent from commonly used landscape pattern metrics by having a low correlation to the other 64 landscape pattern metrics. This landscape metric factor analysis model is useful for both developing landscape metrics, and finding landscape descriptors appropriate for a landscape ecology. By comparing the Fourier landscape pattern metrics ability to explain this dataset we have identified these higher ranking Fourier metrics as capable to describe the landscape. If we choose only the Fourier metrics to interpret the landscape, this analysis has shown that each would be reporting without redundancy. This approach to identifying appropriate landscape pattern metrics for a landscape can be done for any combination of landscape pattern metrics.

These reported Fourier pattern metric values have also shown themselves to be capable to explain general landscape pattern variability by contributing to the explained landscape variability, again with exceptional results from $L S F T$ and $F M F$ respectively contributing $50.06 \%$ and $64.76 \%$ to explained pattern variability. Unlike other landscape pattern metrics (see Neel et al. 2004; Wu, 2004; Wu et al., 2002), this is the Fourier metric that has performed consistently across landscape scale and geographic region (Spivey, 2011). Table 3 shows the best of these independent highly informative metrics. Taken from Table 2, these ten metrics perform generally better for the conterminous United States. Any combination of the resulting landscape pattern metrics in this table are useful for very broad continental scale applications, like long term climate change, or land cover land use forecasting across large scales. We have tested over a wide variety of land cover land use types and these metrics have shown their ability to independently capture relevant landscape variability.

In a separate paper, Spivey \& Vodacek (2017), the relevance of these reliable and unique Fourier landscape pattern metrics for connecting an ecological process to landscape pattern and interpreting the cause is discussed. 
Table 3: The landscape pattern metrics (LPM) ranked by highest expected contribution to the explanation of NLCD 1992 land cover and that are also highly independent metrics. The Fourier metrics are in bold.

\begin{tabular}{cc}
\hline \hline LPM & E.C.C. \\
\hline P005 & $97.46 \%$ \\
FMF & $64.76 \%$ \\
LsFT & $50.06 \%$ \\
BETL & $33.04 \%$ \\
MPFD & $27.32 \%$ \\
OIFT & $25.11 \%$ \\
OEFT & $18.72 \%$ \\
SUMD & $13.55 \%$ \\
ABFT & $13.15 \%$ \\
FMP & $11.81 \%$ \\
\hline
\end{tabular}

\section{REFERENCE}

Anderson, J.R., Hardy, E.E., Roach, J.T., Witmer, R.E. (1976). A land use and land cover classi cation system for use with remote sensor data. Geological survey professional paper 964, U.S. Geological Survey, Washington, D.C., 1976.

Baker, W.L \& Cai, Y. (1992). The r.le programs for multiscale analysis of land-scape structure using the grass geographical information system. Landscape Ecology, 7:291 \{302, 1992.

Detenbeck, N., Johnston, C.A., Niemi, G. (1993). Wetland e ects on lake water quality in the minneapolis/st. paul metropolitan area. Landscape Ecology, 8: 39\{61, 1993.

Frohn, R. (1998). Remote Sensing for Landscape Ecology: New Metric Indicators for Monitoring, Modeling and Assessment of Ecosystems. CRC-Lewis Publishers, Boca Raton, USA, 1998.

Johnes, P., Moss, B., Phillips, G. (1996). The determination of total nitrogen and total phosphorous concentrations in freshwaters from land use, stock headage and population data: Testing a model for use in conservation and water quality management. Freshwater Biology, 36:451\{473, 1996.

Johnson, W.C., Richards, C., Host, G.E., Arthur, J.W. (1997). Landscape in uence on water chemistry in midwestern stream ecosystems. Freshwater Biology, 37 (1):193\{218, 1997.

Jones, K.B., Neale, A.C., Nash, M.S., Van Remortel, R.D., Wickham, J.D., Ritters, K.H., O'Neill, R.V. (2001). Predicting nutrient and sediment loadings to streams from landscape metrics: A multiple watershed study from the united states mid-atlantic region. Landscape Ecology, 16:301\{312, 2001.

Kaushal, S.S., Pace, M.L., Groman, P.M., Band, L.E., Belt, K.T., Mayer, P.M., Welty, C., (2010). Land use and climate variability amplify contaminant pulses. Eos, Transactions, American Geohphysical Union, 91(25):221\{222, July 2010.

Kaushal, S.S., Groman, P.M., Band, L. E., Shields, C.A., Morgan, R.P., Palmer, M.A., Belt, K.T., Swan, C.M., Findlay, S.E.G., Fisher, G.T., (2008). Interac-tion between urbanization and climate variability ampli es watershed nitrate export in maryland. Environmental Science \& Technology, 42(16):5872\{5878, July 2008. 
Klein, R.D. (1979). Urbanization and stream quality impairment. Water Resources Bulletin, 15:948\{963, 1979.

Lovejoy, S. (1982). Area-perimeter relation for rain and cloud areas. Science, 216: 185\{187, 1982.

Magurran, A.E. (1988). Ecological Diversity and its Measurement. Princeton Univer-sity Press, Princeton, NJ, 1988.

McGarigal, K. \& Marks, B.J. (1994). Fragstats: Spatial pattern analysis program for quantifying landscape structure, version 2.0. Oregon State University, Corvallis, 1994.

Neel, Maile C., McGarigal, K., Cushman, Samuel A. (2004). Behavior of class-level landscape metrics across gradients of class aggregation and area. Landscape Ecology, 19:435\{455, 2004.

O'Neill, R.V., Krummel, J.R., Gardner, R.H., Sugihara, G., Jackson, B., DeAngelis, D.L., Milne, B.T., Turner, Monica G., Zygmut, B., Christensen, S.W., Dale, V.H., Graham, R.L. (1988). Indices of landscape pattern. Landscape Ecology, 11:169\{180, 1988.

Osborne, L.L. \& Wiley, M.J. (1988). Empirical relationships between land use/land cover and stream water quality in an agricultural watershed. Journal of Environmental Management, 26:9\{27, 1988.

Pickover, C.A. (1990). Computers, Pattern, Chaos and Beauty, Graphics from an Unseen World. St. Martin's Press, New York, NY, 1990.

Richards, J. \& Jia, X. (1996). Remote Sensing Digital Image Analysis: An Introduction. Springer, 3 edition, 1996.

Ritters, K.H., O'Neill, R. V., Wickham, J. D., Jones, K. B. (1996). A note on contagion metrics of landscape analysis. Landscape Ecology. 11(4): 197-202.

Ritters, K.H., O'Neill, R.V., Hunsaker, C.T., Wickham, J.D., Yankee, D.H., Timmins, S.P., Jones, K.B. and Jackson, B. L., (1995). A factor analysis of landscape pattern and structure metrics. Landscape Ecology, 10:23\{39, 1995.

Schueler, T.R. \& Galli, J. (1992). Environmental impacts of stormwater ponds. In P Kimble and T. Schueler, editors, Watershed Restoration Source Book, page 242. Washington Council Government, 1992.

Spivey, A. (2011). Multiple Scale Landscape Pattern Index Interpretation for the Persistent Monitoring of Land-Cover and Land-Use. Ph.d., Rochester Institute of Technology, 54 Lomb Memorial Drive, May 2011.

Spivey, A. \& Vodacek, A. (2017). Multiscale fourier landscape pattern indices for landscape ecology. In Review, 2017.

Sponseller, R.A., Beneld E.F., Valett, H.M. (2001). Relationships between land use, spatial scale and stream macroinvertebrate communities. Freshwater Biology, 46(10):1409\{1424, 2001.

Vogelmann, J.E., Howard, S.M., Yang, L., Larson, C.R.,. Wylie, B.K, Van Driel, N. (2001). Completion of the 1990s national land cover data set for the conterminous united states from landsat thematic mapper data and ancillary data sources. Photogrammetric Engineering and Remote Sensing, 67:650\{662,2001

Voss, R.F. (1988). Fractals in Nature, chapter From Characterization to Simulation, pages 21\{70. The Science of Fractal Images. Springer-Verlag, New York, NY, 1988.

Wang, L., Lyons, J., Kanehl, P., Gatti, R. (1998). In uences of watershed land use on habitat quality and biotic integrity of wisconsin streams. Fisheries, 22: 6\{12, 1998. 
Weins, J.A. \& Milne, B.T. (1989). Scaling of 'landscapes' in landscape ecology, or, landscape ecology from a beetle's perspective. Landscape Ecology, 3:87\{96, 1989.

Wickham, J.D. \&. Ritters, K.H. (1995). Sensitivity of landscape metrics to pixel size. International Journal of Remtoe Sensing, 16(18):3585\{3594, 1995.

Williams, M.R. \& Melack, John M. (1997). Solute export from forested and partially deforested catchments in the central amazon. Biogeochemistry, 38:67\{102, 1997.

Williams, M.R., Fisher, T.R., Melack, John M. (1997). Solute dynamics in soil wa-ter and groundwater in central amazon catchment undergoing deforestation. Biogeochemistry, 38(3):303\{335, 1997

Woodwell, George M. ed. (1998). Stream Biodiversity; The Ghost of Land Use Past, volume 95, 1998. National Academy of Sciences of the United States.

Wu, J. (20014). Ects of changing scale on landscape pattern analysis: Scaling relations.

Landscape Ecology, 19:125\{138, 2004.

Wu, J., Shen, W., Sun, W., Tueller, P.T. (2002). Empirical patterns of the e ects of changing scale on landscape metrics. Landscape Ecology, 17:761\{782, 2002.

Yuen, S.Y., Fong, Ch.K., Chan, K.L., Leung Y.W. (2004). Frac-tal dimension estimation and noise ltering using hough transform. Signal Processing, 84:907\{917, January 2004. 


\section{APPENDIX}

\section{Landscape Pattern Metrics}

Below lists the metrics used in this analysis. Every Landscape Pattern Metric listed below is taken from, unless otherwise referenced. Independent landscape pattern metrics are numbered, and the indented metrics are highly correlated to their numerated header.

1. Number of LCLU classes

- $\quad$ NTYP (Total number of LCLU classes)

$$
-\quad N T Y P=S
$$

2. Shannon evenness of LCLU classes

- $\quad$ SIDI (Simpson diversity of LCLU classes)

$$
-\quad S I D I=1-\sum_{j=1}^{S} p_{j}^{2}
$$

Here, $S$ is the number of LCLU classes, and $p_{j}$ is the proportion of each

\section{LCLU class $j$.}

- $\quad$ SIEV (Simpson evenness of LCLU classes)

$$
-\quad S I E V=\frac{S I D I}{1-\frac{1}{S}}
$$

Here, $S$ is the number of LCLU classes. See Shannon evenness of LCLU classes for SIDI.

- $\quad$ SHDI (Shannon diversity of attribute classes)

$$
-\quad S H D I=-\sum_{j=1}^{S} p_{j} \ln \left[p_{j}\right]
$$

Here, $S$ is the number of LCLU classes, and $p_{j}$ is the proportion of LCLU

class $j$.

- $\quad$ SHEV (Shannon evenness of LCLU classes)

$$
\text { - } \quad S H E V=\frac{S H D I}{\ln [S]}
$$

Here, $S$ is the number of LCLU classes.

- $\quad$ MCDI (McIntosh diversity of attribute classes )

$$
\text { - } \quad M C D I=\frac{N-\sqrt{\sum_{j=1}^{S} n_{j}^{2}}}{N-\sqrt{N}}
$$

Here, $S$ is the number of LCLU classes, $N$ is the total number of pixels in the landscape, and $n_{j}$ is the total number of pixels in LCLU class $j$.

- $\quad M C E V$ (McIntosh evenness of attribute classes)

$$
-\quad M C E V=\frac{N-\sqrt{\sum_{j=1}^{S} n_{j}^{2}}}{N-\frac{N}{\sqrt{S}}}
$$

Here, $S$ is the number of LCLU classes, $N$ is the total number of pixels in the landscape, and $n_{j}$ is the total number of pixels in LCLU class $j$.

- $\quad$ SHHO (Shannon homogeneity of the adjacency matrix)

$$
\begin{array}{rlr}
S H H O & = & -\sum_{i=1}^{S} \sum_{j=1}^{S} p_{j} \cdot q_{i, j} \ln \left[p_{j} \cdot q_{i, j}\right] \\
& =-\sum_{i=1}^{S} \sum_{j=1}^{S} \frac{n_{i, j}\left[E_{\text {hor }}, E_{\text {vert }}\right]}{N} \ln \left[\frac{n_{i, j}\left[E_{\text {hor }}, E_{\text {vert }}\right]}{N}\right]
\end{array}
$$


Here, $S$ is the number of LCLU classes, $N$ is the total number of pixels in the landscape, and $n_{i, j}\left[E_{\text {hor }}, E_{\text {vert }}\right]$ the total number of horizontal and vertical edge pixels shared by LCLU classes $i$ and $j$.

- $\quad$ SIHO (Simpson homogeneity of adjacency matrix )

$-\quad S I H O=1-\sum_{i=1}^{S} \sum_{j=1}^{S}\left(\frac{n_{i, j}\left[E_{\text {hor }}, E_{\text {vert }}\right]}{N}\right)^{2}$

Here, $S$ is the number of LCLU classes, $N$ is the total number of pixels in the landscape, and $n_{i, j}\left[E_{\text {hor }}, E_{\text {vert }}\right]$ the total number of horizontal and vertical edge pixels shared by LCLU classes $i$ and $j$.

3. Kempton-Taylor Q-statistic

- $\quad K T-Q$ (The inter-quartile slope of the cumulative LCLU abundance curve )

$-\quad K T-Q=\frac{\frac{n_{Q_{1}}}{2}+\frac{n_{Q_{2}}}{2}+\sum_{Q=Q_{1}+1}^{Q_{2}-1} n_{Q}}{\ln \left[\frac{Q_{2}}{Q_{1}}\right]}$,

where $n_{Q}$ is the number of LCLU classes with abundance $Q, Q_{1} ; Q_{2}$ are the $25^{\text {th }}$ and $75^{\text {th }}$ quartiles:

i. $n_{Q_{1}}=n_{j}$ the number of pixels in the LCLU class where

$Q_{1}$ falls - the $1^{s t}-25^{t h}$ percentile number of pixels ii. $n_{Q_{2}}=n_{j}$

the number of pixels in the LCLU class where $Q_{2}$ falls - the

$75^{\text {th }}-100^{\text {th }}$ percentile number of pixels

The quartiles are chosen such that,

and

$$
\sum_{Q=1}^{Q_{1}-1} n_{Q}<\frac{S}{4} \leq \sum_{Q=1}^{Q_{1}} n_{Q},
$$

$$
\sum_{Q=1}^{Q_{2}-1} n_{Q}<\frac{3 \cdot S}{4} \leq \sum_{Q=1}^{Q_{2}} n_{Q} .
$$

where $S$ is the number of LCLU classes.

4. Shannon Contagion

- SHCO (Shannon Contagion)

- $\quad S H C O=1-\frac{S H H O}{2 \cdot \ln [S]}=$ Contagion

Here, $S$ is the number of LCLU classes. See Shannon evenness of LCLU

classes for $\mathrm{SHHO}$.

- $\quad$ SICO (Simpson Contagion)

$$
-\quad S I C O=\frac{S I H O}{1-\frac{1}{S^{2}}}
$$

Here, $S$ is the number of LCLU classes. See Shannon evenness of LCLU classes for $\mathrm{SIHO}$.

5. $\quad$ Sum of LCLU adjacencies

- $\quad S U M D$ (Sum of adjacencies for one LCLU class )

$-\quad S U M D=\sum_{j=1}^{S} n_{j}\left[E_{h}, E_{v}\right]$

Here, $S$ is the number of LCLU classes, and $n_{j}\left[E_{h}, E_{v}\right]$ are the total

horizontal and vertical edges of LCLU class $j$.

- TENT (Average fractal estimator of LCLU class configurational entropy from the scaling of LCLU class density to the size of the neighborhood of an arbitrary pixel in the class) 
- $\quad P_{t}\left[L ; n_{j}\right]$ is the probability of finding $t$ other pixels of the same LCLU class, in an $L \times L$ kernel, centered on an arbitrarily chosen pixel.

$$
P_{t}\left[L ; n_{j}\right]=\frac{s_{t, L}[j]}{n_{j}}
$$

where $s_{t, L}[j]$ is the number of $L \times L$ kernels that had $t$ pixels of the same LCLU class $j$, and $n_{j}$ is the number of pixels in LCLU class $j$.

Letting $n_{j}[L]$ be the maximum number of pixels, of the same LCLU class $j$, observed in any $L \times L$ kernel:

$$
M^{0}\left[P_{t}\left[L ; n_{j}\right]\right]=\sum_{t=1}^{n_{j}[L]} \ln [t] \cdot P_{t}\left[L ; n_{j}\right]
$$

From each LCLU class with $>400$ pixels, a random sample of at least 400 pixels are selected. Square kernels of size $L=5,15,25,35$, and 45 are placed around each sampled pixel and the occurrence of pixels of the same LCLU class are counted for each kernel size. Letting $\beta_{10 j}$ be the estimated slope from the regression of $M^{0}\left[P_{t}\left[L ; n_{j}\right]\right]$ on $\ln [L]$ for the $j^{\text {th }} \operatorname{LCLU}$ class, the fractal estimator is:

$$
T E N T=\sum_{j=1}^{S} \frac{n_{j}}{N} \beta_{10 j}
$$

Here, $S$ is the number of LCLU classes, $N$ is the total number of pixels in the landscape, and $n_{j}$ is the total number of pixels in LCLU class $j$.

- TMAS (Fractal estimator of LCLU class mass from the scaling of LCLU class density to the size of a neighborhood of an arbitrary pixel in the class.)

- $\quad P_{t}\left[L ; n_{j}\right]$ is the probability of finding $t$ other pixels of the same LCLU class, in an $L \times L$ kernel, centered on an arbitrarily chosen pixel.

$$
P_{t}\left[L ; n_{j}\right]=\frac{s_{t, L}[j]}{n_{j}}
$$

where $s_{t, L}[j]$ is the number of $L \times L$ kernels that had $t$ pixels of the same LCLU class $j$, and $n_{j}$ is the number of pixels in LCLU class $j$.

Letting $n_{j}[L]$ be the maximum number of pixels, of the same LCLU class $j$, observed in any $L \times L$ kernel:

$$
M^{1}\left[P_{t}\left[L ; n_{j}\right]\right]=\sum_{t=1}^{n_{j}[L]} t \cdot P_{t}\left[L ; n_{j}\right]
$$

From each LCLU class with $>400$ pixels, a random sample of at least 400 pixels are selected. Square kernels of size $L=5,15,25,35$, and 45 are placed around each sampled pixel and the occurrence of pixels of the same LCLU class are counted for each kernel size. Letting $\beta_{11 j}$ be the estimated slope from the regression of $\ln \left[M^{1}\left[P_{t}\left[L ; n_{j}\right]\right]\right]$ on $\ln [L]$ for the $j^{\text {th }}$ LCLU class. The fractal estimator is:

$$
T M A S=\sum_{j=1}^{S} \frac{n_{j}}{N} \beta_{11 j}
$$

Here, $S$ is the number of LCLU classes, $N$ is the total number of pixels in the landscape, and $n_{j}$ is the total number of pixels in LCLU class $j$.

- TVAR (Fractal estimator of LCLU class variance from the scaling of LCLU class density to the size of the neighborhood of an arbitrary pixel in the class)

- $\quad P_{t}\left[L ; n_{j}\right]$ is the probability of finding $t$ other pixels of the same LCLU class, in an $L \times L$ kernel, centered on an arbitrarily chosen pixel.

$$
P_{t}\left[L ; n_{j}\right]=\frac{s_{t, L}[j]}{n_{j}}
$$

where $s_{t, L}[j]$ is the number of $L \times L$ kernels that had $t$ pixels of the same LCLU class $j$, and $n_{j}$ is the number of pixels in LCLU class $j$. 
Letting $n_{j}[L]$ be the maximum number of pixels, of the same LCLU class $j$, observed in any $L \times L$ kernel:

$$
M^{2}\left[P_{t}\left[L ; n_{j}\right]\right]=\sum_{t=1}^{n_{j}[L]} t^{2} P_{t}\left[L ; n_{j}\right]
$$

From each LCLU class with $>400$ pixels, a random sample of at least 400 pixels are selected. Square kernels of size $L=5,15,25,35$, and 45 are placed around each sampled pixel and the occurrence of pixels of the same LCLU class are counted for each kernel size. Letting $\beta_{12 j}$ be the estimated slope from the regression of $\ln \left[M^{2}\left[P_{t}\left[L ; n_{j}\right]\right]\right]$ on $\ln (L)$ for the $j^{t h}$ LCLU class. The fractal estimator is:

$$
T V A R=\sum_{j=1}^{S} \frac{n_{j}}{N} \beta_{12 j}
$$

Here, $S$ is the number of LCLU classes, $N$ is the total number of pixels in the landscape, and $n_{j}$ is the total number of pixels in LCLU class $j$.

- $\quad P 050$ (Weighted average proportion of pixels contained in patches with area $>50$ pixels)

$$
-\quad P 050=\sum_{j=1}^{S} \frac{n_{j}}{N} \frac{\sum_{\tau=1}^{\psi_{j}} n_{\tau}^{*}}{\sum_{\tau=1}^{\psi_{j}} n_{\tau}}
$$

where $S$ is the number of LCLU classes, $N$ is the total number of pixels in the landscape, $n_{j}$ is the total number of pixels in the LCLU class $j, \psi_{j}$ is the total number of nearest-neighbor patches of LCLU class $j$, and $n_{\tau}$ is the total number of pixels in patch $\tau$.

$$
n_{\tau}^{*}=\left\{\begin{array}{l}
0 \text { if } n_{\tau}<51, \\
n_{\tau} \text { otherwise. }
\end{array}\right.
$$

- $\quad P 500$ (Weighted average proportion of pixels contained in patches with area $>500$ pixels)

$$
-\quad P 500=\sum_{j=1}^{S} \frac{n_{j}}{N} \frac{\sum_{\tau=1}^{\psi_{j}} n_{\tau}^{*}}{\sum_{\tau}^{\psi_{j}} n_{\tau}}
$$

where $S$ is the number of LCLU classes, $N$ is the total number of pixels in the landscape, $n_{j}$ is the total number of pixels in the LCLU class $j, \psi_{j}$ is the total number of nearest-neighbor patches of LCLU class $j$, and $n_{\tau}$ is the total number of pixels in patch $\tau$.

$$
n_{\tau}^{*}=\left\{\begin{array}{l}
0 \text { if } n_{\tau}<501 \\
n_{\tau} \text { otherwise }
\end{array}\right.
$$

6. $\quad$ Average LCLU class lacunarity

- $\quad T L A C$ (Average LCLU class lacunarity from the scaling of class density with kernel size)

$$
-\quad T L A C=T V A R-T E N T
$$

See, Sum of LCLU adjacencies

7. $\quad$ Average proportion of area in patches larger than 5 pixels

- $\quad P 005$ (Weighted average proportion of pixels contained in patches with area $>5$ pixels-Jackson's Contagion statistic )

$$
-\quad P 005=\sum_{j=1}^{S} \frac{n_{j}}{N} \frac{\sum_{\tau=1}^{\psi_{j}} n_{\tau}^{*}}{\sum_{\tau}^{\psi_{j}} n_{\tau}}
$$

where $S$ is the number of LCLU classes, $N$ is the total number of pixels in the landscape, $n_{j}$ is the total number of pixels in the LCLU class $j, \psi_{j}$ is the total 
number of nearest-neighbor patches of LCLU class $j$, and $n_{\tau}$ is the total number of pixels in patch $\tau$.

$$
n_{\tau}^{*}=\left\{\begin{array}{l}
0 \text { if } n_{\tau}<6, \\
n_{\tau} \text { otherwise. }
\end{array}\right.
$$

8. $\quad$ Perimeter-area scaling, patch perimeter complexity

- $\quad O E F C$ (Fractal estimator of patch perimeter complexity from perimeter-area scaling enclosing edges basis )

$-\quad O E F C=2 \cdot \beta_{1}$

where $\beta_{1}$ is the estimated slope from the regression of the natural log of left-diagonal and right-diagonal patch edge pixels, $\ln \left[n_{\tau}\left[E_{\text {left }}, E_{\text {right }}\right]\right]$, on the natural logarithm of the total number of pixels in LCLU patch $\tau$, $\ln \left[n_{\tau}\right]$, for all nearest-neighbor patches greater than 3 pixels, $n_{\tau}>3$, that do not touch the border of the LCLU map.

- $\quad$ OIFC (Fractal estimator of patch perimeter complexity from perimeter-area scaling, all edges basis)

$$
O I F C=2 \cdot \beta_{3}
$$

where $\beta_{3}$ is the estimated slope from the regression of left-diagonal, right-diagonal, horizontal, and vertical patch edge pixels,

$\ln \left[n_{\tau}\left[E_{\text {left }}, E_{\text {right }}, E_{\text {hor }}, E_{\text {vert }}\right]\right]$, on the natural logarithm of the total number of pixels in LCLU patch $\tau, \ln \left[n_{\tau}\right]$, for all nearest-neighbor patches greater than 3 pixels, $n_{\tau}>3$, that do not touch the border of the LCLU map.

- $\quad$ OCFC (Fractal estimator of patch perimeter complexity from perimeter-area scaling, enclosing pixels basis)

- $O C F C=2 \cdot \beta_{2}$

where $\beta_{2}$ is the estimated slope from the regression of the left-diagonal, right-diagonal, horizontal, and vertical patch edge pixels complement,

$$
\ln \left[n_{\tau}-n_{\tau}\left[E_{\text {left }}, E_{\text {right }}, E_{\text {hor }}, E_{\text {vert }}\right]\right]=\ln \left[n_{\tau}^{\mathrm{C}}\right]
$$

on the natural logarithm of the total number of pixels in LCLU patch $\tau$, $\ln \left[n_{\tau}\right]$, for all nearest-neighbor patches greater than 3 pixels, $n_{\tau}>3$, that do not touch the border of the LCLU maps.

9. Perimeter-area scaling, patch topology transformation, enclosing pixel basis

- $\quad$ OEFT Fractal estimator of patch topology from perimeter-area scaling, enclosing edges basis

$$
O E F T=\frac{1}{\beta_{1}}
$$

where $\beta_{1}$ is the estimated slope from the regression of the natural log of left-diagonal and right-diagonal patch edge pixels, $\ln \left[n_{\tau}\left[E_{\text {left }}, E_{\text {right }}\right]\right]$, on the natural logarithm of the total number of pixels in LCLU patch $\tau$, $\ln \left[n_{\tau}\right]$, for all nearest-neighbor patches greater than 3 pixels, $n_{\tau}>3$, that do not touch the border of the LCLU map.

- $\quad$ OIFT Fractal estimator of patch topology from perimeter-area scaling, all edges basis.

$$
\text { - OIFT }=\frac{1}{\beta_{3}}
$$

where $\beta_{3}$ is the estimated slope from the regression of left-diagonal, right-diagonal, horizontal, and vertical patch edge pixels, $\ln \left[n_{\tau}\left[E_{\text {left }}, E_{\text {right }}, E_{\text {hor }}, E_{\text {vert }}\right]\right]$, on the natural logarithm of the total number of pixels in LCLU patch $\tau, \ln \left[n_{\tau}\right]$, for all nearest-neighbor 
patches greater than 3 pixels, $n_{\tau}>3$, that do not touch the border of the LCLU map.

- $\quad$ OCFT Fractal estimator of patch topology from perimeter-area scaling, enclosing pixels basis.

OCFT $=\frac{1}{\beta_{2}}$

where $\beta_{2}$ is the estimated slope from the regression of the left-diagonal, right-diagonal, horizontal, and vertical patch edge pixels complement,

$$
\ln \left[n_{\tau}-n_{\tau}\left[E_{\text {left }}, E_{\text {right }}, E_{\text {hor }}, E_{\text {vert }}\right]\right]=\ln \left[n_{\tau}^{C}\right]
$$

on the natural logarithm of the total number of pixels in LCLU patch $\tau$, $\ln \left[n_{\tau}\right]$, for all nearest-neighbor patches greater than 3 pixels, $n_{\tau}>3$, that do not touch the border of the LCLU maps.

10. Patch area-bounding circle scaling

- $\quad A B F T$ (Fractal estimator of patch topology from area-bounding rectangular kernel scaling)

$-\quad A B F T=\beta_{4}$

where $\beta_{4}$ is the estimated slope from the regression of the natural logarithm of the total number of pixels in the LCLU patch $\tau, \ln \left(n_{\tau}\right)$, on the natural logarithm of the largest rectangular kernel side length difference $Y_{\tau}$ from a box bounding a patch $\tau$ is:

$$
Y_{\tau}=\ln \left[\operatorname{MAX}\left(L_{\text {hor }, \tau}, L_{\text {vert }, \tau}\right)\right]
$$

Here, $L_{\text {hor }, \tau} \times L_{\text {vert }, \tau}$ is the rectangular kernel size that fits over patch $\tau$. This is for all nearest-neighbor patches greater than 3 pixels, $n_{\tau}>3$, that do not touch the border of the LCLU maps.

11. Patch perimeter complexity from the scaling of Euclidean distance to actual distance along large patch perimeters

- $\quad$ BETL (Fractal estimator of perimeter complexity from scaling the average patch edge to variable square kernel size )

$$
B E T L=\frac{1}{\widehat{\psi}} \sum_{\hat{\tau}=1}^{\widehat{\psi}} \frac{1}{\beta_{6 \hat{\tau}}}
$$

where $\beta_{6 \hat{\tau}}$ is the estimated box-counting fractal dimension of the $\hat{\tau}^{t h}$ patch — only for patches with area $n_{\tau}>400$ —using the average Euclidean distance $\bar{r}_{\hat{\tau}}$ from patch $\hat{\tau}$ centroid $\left(\bar{x}_{\hat{\tau}}, \bar{y}_{\hat{\tau}}\right)$ to a $L \times L$ patch bounding-box, or patch perimeter (whichever comes first) regression, $\ln \left[\bar{r}_{\hat{\tau}}\right]$ on the natural logarithm of bounding-box perimeter, perimeter $=$ $4 \cdot L$, or patch $\hat{\tau}$, perimeter $=n_{\hat{\tau}}\left[E_{\text {left }}, E_{\text {right }}, E_{\text {hor }}, E_{\text {vert }}\right]$, perimeter $\ln [$ perimeter $]$.

Ritters describes the procedures as follows. For each patch meeting the minimum 400 pixel area $\left(n_{\hat{\tau}}>400\right)$ constraint, the average Euclidean distance $\bar{r}_{\tau}$ along the perimeter is found for actual distances $L=4,8,16$, $32,64,128$, and 512 bounding-box pixels. The proportions of patch perimeters that touched the map border are excluded.

The expression for average patch centroid Euclidean distance is:

$$
\bar{r}_{\hat{\tau}}=\frac{1}{n_{\hat{\tau}}} \sum_{\hat{\tau}=1}^{\widehat{\psi}} r_{\hat{\tau}}
$$

where the patch $\hat{\tau}$ distances $r_{\hat{\tau}}$ are for all $\hat{\tau}=1,2,3 \ldots \widehat{\psi}$ pixels, and are normalized by the $\hat{\tau}$ patch area $n_{\hat{\tau}}$.

Within patch distances are expressed as: 


$$
r_{\hat{\tau}}=\sqrt{\sum_{u} \sum_{v}\left(x_{u}-\bar{x}_{\hat{\tau}}\right)^{2}+\left(y_{v}-\bar{y}_{\hat{\tau}}\right)^{2}}
$$

for every pixel $u=v=1,2,3,4 \ldots n_{\hat{\tau}}$ within patch $\hat{\tau}$, for the total number of nearest-neighbor patches $\widehat{\psi}$ patches.

12. Metric of large-patch 'mass' from the scaling of patch density with neighborhood

$\underline{\text { size }}$

- $\quad P D$ (Patch density. The number of patches (units: $\mathrm{km}^{-2}$ ))

$-\quad P D=\frac{\psi}{N_{S}\left(\mathrm{~km}^{2}\right)}$

where $\psi$ is the total number of nearest-neighbor patches, and $N_{s}$ is the total number of pixels in a square landscape (units: $\mathrm{km}^{2}$ ).

- $\quad$ PENT (Fractal estimator of patch configurational entropy from the scaling of patch density to the size of a neighborhood of an arbitrary pixel in the patch )

- $\quad P_{t}\left[L ; n_{\tau}^{*}\right]$ is the probability of finding $t$ other pixels of the same LCLU class, in an $L \times L$ kernel, centered on an arbitrarily chosen pixel. For example, $P_{2}\left[L=5 ; n_{\tau}^{*}\right]$ is the probability of finding two more pixels of a patch in a $5 \times 5$ square centered on an arbitrary pixel in the patch. In general,

$$
P_{t}\left[L ; n_{\tau}^{*}\right]=\frac{s_{t, L}}{n_{\tau}^{*}}
$$

where $s_{t, L}$ is the number of $L \times L$ kernels that had $t$ pixels of the same LCLU class, and $n_{j}$ is the number of pixels in LCLU class $j$.

Letting $n_{j}[L]$ be the maximum number of pixels of the same LCLU class $j$, observed in any $L \times L$ kernel:

$$
M^{0}\left[P_{t}\left[L ; n_{\tau}^{*}\right]\right]=\sum_{t=1}^{n_{j}[L]} \ln [t] \cdot P_{t}\left[L ; n_{\tau}^{*}\right]
$$

From each patch with size $>400$ pixels, a random sample of at least 400 pixels was selected.

$$
n_{\tau}^{*}=\left\{\begin{array}{l}
0 \text { if } n_{\tau}<401, \\
n_{\tau} \text { otherwise }
\end{array}\right.
$$

Square kernels of size $L=5,15,25,35$, and 45 are placed around each sampled pixel, and the occurrences of pixels of the same patch were counted for each kernel size. The values of $P_{t}\left[L ; n_{\tau}^{*}\right]$ and $M^{0}\left[P_{t}\left[L ; n_{\tau}^{*}\right]\right]$ were calculated after the counts are accumulated for all sampled pixels. Letting $\beta_{7 \hat{\tau}}$ be the estimated slope from the regression of $M^{0}\left[P_{t}\left[L ; n_{\tau}^{*}\right]\right]$ on $\ln [L]$ for the $\hat{\tau}^{\text {th }}$ patch. The fractal estimator is:

$$
P E N T=\frac{1}{\widehat{\psi}} \sum_{\widehat{\tau}=1}^{\widehat{\psi}} \beta_{7 \hat{\tau}}
$$

where, $\widehat{\psi}$ is the total number of nearest-neighbor patches.

- $\quad$ PMAS (Fractal estimator of patch mass from the scaling of patch density to the size of a neighborhood of an arbitrary cell in the patch )

- $P_{t}\left[L ; n_{\tau}^{*}\right]$ is the probability of finding $t$ other pixels of the same LCLU class, in an $L \times L$ kernel, centered on an arbitrarily chosen pixel. For example, $P_{2}\left[L=5 ; n_{\tau}^{*}\right]$ is the probability of finding two more pixels of a patch in a $5 \times 5$ square centered on an arbitrary pixel in the patch. In general,

$$
P_{t}\left[L ; n_{\tau}^{*}\right]=\frac{s_{t, L}}{n_{\tau}^{*}}
$$

where $s_{t, L}$ is the number of $L \times L$ kernels that had $t$ pixels of the same LCLU class, and $n_{j}$ is the number of pixels in LCLU class $j$.

Letting $n_{j}[L]$ be the maximum number of pixels of the same LCLU class $j$, observed in any $L \times L$ kernel: 


$$
M^{1}\left[P_{t}\left[L ; n_{\tau}^{*}\right]\right]=\sum_{t=1}^{n_{j}[L]} t \cdot P_{t}\left[L ; n_{\tau}^{*}\right]
$$

From each patch with size $>400$ pixels, a random sample of at least 400 pixels was selected.

$$
n_{\tau}^{*}=\left\{\begin{array}{l}
0 \text { if } n_{\tau}<401 \\
n_{\tau} \text { otherwise }
\end{array}\right.
$$

Square kernels of size $L=5,15,25,35$, and 45 are placed around each sampled pixel, and the occurrences of pixels of the same patch were counted for each kernel size. The values of $P_{t}\left[L ; n_{\tau}^{*}\right]$ and $M^{1}\left[P_{t}\left[L ; n_{\tau}^{*}\right]\right]$ were calculated after the counts are accumulated for all sampled pixels. Letting $\beta_{8 \hat{\tau}}$ be the estimated slope from the regression of $M^{1}\left[P_{t}\left[L ; n_{\tau}^{*}\right]\right]$ on $\ln [L]$ for the $\hat{\tau}^{\text {th }}$ patch. The fractal estimator is:

$$
P M A S=\frac{1}{\widehat{\psi}} \sum_{\hat{\tau}=1}^{\widehat{\psi}} \beta_{8 \hat{\tau}}
$$

where, $\widehat{\psi}$ is the total number of nearest-neighbor patches.

- $\quad$ PVAR (Fractal estimator of patch variance from the scaling of patch density to the size of a neighborhood of an arbitrary pixel in the patch )

- $\quad P_{t}\left[L ; n_{\tau}^{*}\right]$ is the probability of finding $t$ other pixels of the same LCLU class, in an $L \times L$ kernel, centered on an arbitrarily chosen pixel. For example,

$P_{2}\left[L=5 ; n_{\tau}^{*}\right]$ is the probability of finding two more pixels of a patch in a $5 \times 5$ square centered on an arbitrary pixel in the patch. In general,

$$
P_{t}\left[L ; n_{\tau}^{*}\right]=\frac{s_{t, L}}{n_{\tau}^{*}}
$$

where $s_{t, L}$ is the number of $L \times L$ kernels that had $t$ pixels of the same LCLU class, and $n_{j}$ is the number of pixels in LCLU class $j$.

Letting $n_{j}[L]$ be the maximum number of pixels of the same LCLU class $j$, observed in any $L \times L$ kernel:

$$
M^{2}\left[P_{t}\left[L ; n_{\tau}^{*}\right]\right]=\sum_{t=1}^{n_{j}[L]} t^{2} \cdot P_{t}\left[L ; n_{\tau}^{*}\right]
$$

From each patch with size $>400$ pixels, a random sample of at least 400 pixels was selected.

$$
n_{\tau}^{*}=\left\{\begin{array}{l}
0 \text { if } n_{\tau}<401, \\
n_{\tau} \text { otherwise }
\end{array}\right.
$$

Square kernels of size $L=5,15,25,35$, and 45 are placed around each sampled pixel, and the occurrences of pixels of the same patch were counted for each kernel size. The values of $P_{t}\left[L ; n_{\tau}^{*}\right]$ and $M^{2}\left[P_{t}\left[L ; n_{\tau}^{*}\right]\right]$ were calculated after the counts are accumulated for all sampled pixels. Letting $\beta_{9 \hat{\tau}}$ be the estimated slope from the regression of $M^{2}\left[P_{t}\left[L ; n_{\tau}^{*}\right]\right]$ on $\ln [L]$ for the $\hat{\tau}^{\text {th }}$ patch. The fractal estimator is:

$$
P E N T=\frac{1}{\widehat{\psi}} \sum_{\hat{\tau}=1}^{\widehat{\psi}} \beta_{9 \hat{\tau}}
$$

where, $\widehat{\psi}$ is the total number of nearest-neighbor patches.

13. Average large-patch lancunarity from the scaling from the scaling of patch density with neighborhood size

- $\quad P L A C$ (Average large-patch lancunarity)

- $\quad P L A C=$ PVAR - PENT

See Metric of large-patch 'mass' from the scaling of patch density with neighborhood size.

14. Number of patches

- NPAT (Number of patches) 
$-\quad N P A T=\psi$

where $\psi$ is the number of nearest-neighbor patches in the LCLU map.

15. Largest patch index

- $\quad$ LPI (The ratio of area of the largest patch to the total area of the landscape (unit: \%))

$$
-L P I=\frac{\operatorname{MAX}\left[n_{\tau}\right]}{N}
$$

where $N$ is the total number of pixels in the landscape, and $n_{\tau}$ is the total number of pixels in nearest-neighbor patch $\tau$.

16. $\quad$ Average patch size or area

- $\quad$ PSIZ (Average patch size or area )

$$
-\quad P S I Z=\frac{1}{\psi} \sum_{\tau=1}^{\psi} n_{\tau}
$$

where $\psi$ is the total number of nearest-neighbor patches, and $n_{\tau}$ is the total area of patch $\tau$.

17. Patch size coefficient of variation

- $\quad$ PSCV (The standard deviation of patch size divided by mean patch size for the entire landscape (unit:\%))

$$
\text { - } \quad P S C V=\frac{P S S D}{P S I Z}
$$

See Average patch size or area for PSIZ and Patch size standard deviation for PSSD.

18. Total edge

- $T E$ (The sum of the lengths of all edge segments (unit: $m$ ))

$-\quad T E=E_{\text {left }}+E_{\text {right }}+E_{\text {hor }}+E_{\text {vert }}$

Here, the left-diagonal, right-diagonal, horizontal, and vertical LCLU map edge pixels are $E_{\text {left }}, E_{\text {right }}, E_{\text {hor }}$, and $E_{\text {vert }}$ respectively.

19. Average patch radius of gyration

- $O E D G$ (Average number of edges enclosing a patch-'outside edges')

- $\quad O E D G=\frac{1}{\psi} \sum_{\tau=1}^{\psi} n_{\tau}\left[E_{\text {left }}, E_{\text {right }}\right]$

where $\psi$ is the total number of nearest-neighbor patches, and $n_{\tau}\left[E_{\text {left }}, E_{\text {right }}\right]$ are left-diagonal and right-diagonal patch $\tau$ edge pixels.

- $\quad T E D G$ (Average total number of perimeter edges, or 'perimeter length', per patch)

$T E D G=I E D G+O E D G$

See Average number of inside edges per patch for IEDG and Average patch radius of gyration for $O E D G$.

- $\quad$ OPER (Average number of pixels enclosing a patch-'outside pixels')

- $\quad$ OPER $=\frac{1}{\psi} \sum_{\tau=1}^{\psi} n_{\tau}^{\mathrm{C}}$

where $\psi$ is the total number of nearest neighbor patches, and all patch $\tau$ nearest-neighbor pixels that are not left-diagonal, right- diagonal, horizontal, or vertical edge pixels are $n_{\tau}^{C}=\left(n_{\tau}-n_{\tau}\left[E_{\text {left }}, E_{\text {right }}, E_{\text {hor }}, E_{\text {vert }}\right]\right)$.

- $\quad R G Y R$ (Average radius of gyration)

$R G Y R=\frac{1}{\psi} \sum_{\tau=1}^{\psi} r_{\tau}$

The patch centroid $\left(\bar{x}_{\tau}, \bar{y}_{\tau}\right)$ is used to find the radius of gyration $r_{\tau}$ of patch $\tau$ :

$$
r_{\tau}=\sqrt{\sum_{u} \sum_{v}\left(x_{u}-\bar{x}_{\tau}\right)^{2}+\left(y_{v}-\bar{y}_{\tau}\right)^{2}}
$$


for every pixel $u+v=2,3,4 \ldots n_{\tau}$ within nearest-neighbor patch $\tau$, for the total number of nearest-neighbor patches $\psi$.

- $\quad L O A X$ (Average length of long axis)

- $\quad L O A X=\frac{1}{\psi} \sum_{\tau=1}^{\psi} L_{h o r, \tau}$

where $\psi$ is the total number of nearest-neighbor patches, and $L_{h o r, \tau}$ is the longest horizontal diameter of patch $\tau$.

20. Average number of inside edges per patch

- $\quad I E D G$ (Average number of edges between a patch and its inclusions - inside edges')

$-\quad I E D G=\frac{1}{\psi} \sum_{\tau=1}^{\psi} n_{\tau}\left[E_{\text {hor }}, E_{\text {vert }}\right]$

where $\psi$ is the total number of nearest-neighbor patches, and $n_{\tau}\left[E_{\text {hor }}, E_{\text {vert }}\right]$ are horizontal and vertical patch $\tau$ edge pixels.

21. Landscape shape index

- $\quad$ LSI (A modified perimeter-area ratio)

$-\quad L S I=\frac{0.25 \cdot n_{\tau}[T E]}{\sqrt{N}}$

where $N$ is the total number of pixels in the landscape, and $n_{\tau}[T E]$ is the total number of nearest-neighbor patch edges (unitless). See Average patch radius of gyration for $T E$.

22. Mean patch shape index

- $\quad$ MSI (A patch-level shape index averaged over all patches in the landscape)

$-\quad M S I=\frac{1}{\psi} \sum_{\tau=1}^{\psi} \frac{0.25 \cdot n_{\tau}[T E]}{\sqrt{n_{\tau}}}$

where $\psi$ is the total number of nearest-neighbor patches, $n_{\tau}$ is the total area of patch $\tau$, and $n_{\tau}[T E]$ is the total number of patch edges (unitless). See Average patch radius of gyration for $T E$.

23. Area-Weighted mean patch shape index

- $\quad A W M S I$ (Mean patch shape index weighted by relative patch size)

$A W M S I=\sum_{\tau=1}^{\psi} \frac{0.25 \cdot n_{\tau}[T E]}{\sqrt{n_{\tau}}} \cdot \frac{n_{\tau}}{N}$

where $N$ is the total number of pixels in the landscape, $\psi$ is the total number of nearest-neighbor patches, $n_{\tau}$ is the total area of patch $\tau$, and $n_{\tau}[T E]$ is the total number of patch edges (unitless). See Average patch radius of gyration for $T E$.

24. Double-Log fractal dimension

- $\quad D L F D$ (The fractal dimension of the entire landscape)

- Twice the inverse regression line slope between the logarithm of patch area and the logarithm of patch perimeter:

$D L F D=2 \cdot\left(\frac{\psi \cdot \sum_{\tau=1}^{\psi} \ln \left[n_{\tau}[T E]\right] \ln \left[n_{\tau}\right]-\sum_{\tau=1}^{\psi} \ln \left[n_{\tau}\right]}{\psi \cdot \sum_{\tau=1}^{\psi} \ln \left[n_{\tau}[T E]^{2}\right]-\left(\sum_{\tau=1}^{\psi} \ln \left[n_{\tau}[T E]\right]^{2}\right)}\right)^{-1}$

where $\psi$ is the total number of nearest-neighbor patches, $n_{\tau}$ is the total area of patch $\tau$, and $n_{\tau}[T E]$ is the total number of patch edges (unitless). See Average patch radius of gyration for $T E$.

25. Mean patch fractal dimension

- $\quad M P F D$ (The average fractal dimension of individual patches in the landscape)

The summation of fractal dimension for all patches divided by the total number of patches in the landscape:

$M P F D=\frac{1}{\psi} \sum_{\tau=1}^{\psi} \frac{2 \cdot \ln \left[0.25 \cdot n_{\tau}[T E]\right]}{\ln \left[n_{\tau}\right]}$ 
where $\psi$ is the total number of nearest-neighbor patches, $n_{\tau}$ is the total area of patch $\tau$, and $n_{\tau}[T E]$ is the total number of patch edges (unitless). See Average patch radius of gyration for $T E$.

26. Area-weighted mean patch fractal dimension

- $\quad A W M P F D$ (The patch fractal dimension weighted by relative patch area)

$-\quad A W M P F D=\sum_{\tau=1}^{\psi} \frac{2 \cdot \ln \left[0.25 \cdot n_{\tau}[T E]\right]}{\ln \left[n_{\tau}\right]} \cdot \frac{n_{\tau}}{N}$

where $N$ is the total number of pixels in the landscape, $\psi$ is the total number of nearest-neighbor patches, $n_{\tau}$ is the total area of patch $\tau$, and $n_{\tau}[T E]$ is the total number of patch edges (unitless). See Average patch radius of gyration for $T E$.

27. $\quad$ Square pixel index

- $\quad S q P$ (A normalized perimeter-area ratio )

$-\quad S q P=1-\frac{4 \sqrt{N}}{(T E)}$

where $N$ is the total number of pixels in the landscape, and $T E$ are all left-diagonal, right-diagonal, horizontal, and vertical edge pixels in the landscape (unitless). See Average patch radius of gyration for TE.

28. Average patch perimeter-area ratio

- $\quad P A-1$ (Average nearest-neighbor perimeter-area ratio)

$-\quad P A-1=\frac{1}{\psi} \sum_{\tau=1}^{\psi} \frac{n_{\tau}\left[E_{\text {left }}, E_{\text {right }}\right]}{n_{\tau}}$

where $\psi$ is the total number of nearest-neighbor patches, $n_{\tau}$ is the total area of patch $\tau$, and $n_{\tau}\left[E_{\text {left }}, E_{\text {right }}\right]$ are left-diagonal and right-diagonal patch $\tau$ edge pixels.

29. $\quad$ Average patch adjusted perimeter-area ratio

- $\quad P A-2$ (Average adjusted perimeter-area ratio )

- $\quad P A-2=\frac{1}{\psi} \sum_{\tau=1}^{\psi} \frac{0.282 \cdot n_{\tau}\left[E_{\text {left }}, E_{\text {right }}\right]}{\sqrt{n_{\tau}}}$

where $\psi$ is the total number of nearest-neighbor patches, $n_{\tau}$ is the total area of patch $\tau$, and $n_{\tau}\left[E_{\text {left }}, E_{\text {right }}\right]$ are left-diagonal and right-diagonal patch $\tau$ edge pixels.

30. Average patch normalized area, square model

- $\quad$ NACI (Average normalized area, circular model)

- NACI $=\frac{1}{\psi} \sum_{\tau=1}^{\psi} \frac{4 \pi \cdot n_{\tau}}{n_{\tau}\left[E_{\text {left }}, E_{\text {right }}\right]^{2}}$

where $\psi$ is the total number of nearest-neighbor patches, $n_{\tau}$ is the total area of patch $\tau$, and $n_{\tau}\left[E_{\text {left }}, E_{\text {right }}\right]$ are left-diagonal and right-diagonal patch $\tau$ edge pixels.

- $\quad$ NASQ (Average normalized area, square model)

- $\quad N A S Q=\frac{1}{\psi} \sum_{\tau=1}^{\psi} \frac{16 \cdot n_{\tau}}{n_{\tau}\left[E_{\text {left }}, E_{\text {right }}\right]^{2}}$

where $\psi$ is the total number of nearest-neighbor patches, $n_{\tau}$ is the total area of patch $\tau$, and $n_{\tau}\left[E_{\text {left }}, E_{\text {right }}\right]$ are left-diagonal and right-diagonal patch $\tau$ edge pixels.

- $\quad$ BRRA (Average bounding rectangle ratio)

$-\quad B R R A=\frac{1}{\psi} \sum_{\tau=1}^{\psi} \frac{n_{\tau}}{\chi_{\tau}}$

where

$$
\chi_{\tau}=L_{\text {hor }, \tau} \times L_{\text {vert }, \tau}
$$


Here, $\psi$ is the total number of nearest-neighbor patches, $n_{\tau}$ is the total area of patch $\tau, L_{h o r, \tau}$ and $L_{v e r t, \tau}$ are respectively the rectangular kernel horizontal and vertical sides that fit over patch $\tau$.

31. Average patch topology ratio

- $\quad$ NFTD (Average topology ratio)

$-\quad N F T D=\frac{1}{\psi} \sum_{\tau=1}^{\psi} 2-\frac{\left(n_{\tau}^{C}-4 \sqrt{n_{\tau}}\right)}{\left(2 \cdot n_{\tau}+2-4 \sqrt{n_{\tau}}\right)}$

where $\psi$ is the total number of nearest-neighbor patches, and $n_{\tau}$ is the total area of patch $\tau$

32. Average patch ratio of number of inside edges of area

- PORO (Average ratio of number of inside edges to area)

- PORO $=\frac{1}{\psi} \sum_{\tau=1}^{\psi} \frac{n_{\tau}\left[E_{\text {hor }}, E_{\text {vert }}\right]}{n_{\tau}}$

where $\psi$ is the total number of nearest-neighbor patches, $n_{\tau}$ is the total area of patch $\tau$, and $n_{\tau}\left[E_{\text {hor }}, E_{\text {vert }}\right]$ are horizontal and vertical patch $\tau$ edge pixels.

33. Average patch adjusted perimeter-area ratio

- $\quad$ DSTA (Average adjusted area-perimeter ratio-Gardner's D-statistic)

- DSTA $\left.=\frac{1}{\psi} \sum_{\tau=1}^{\psi} \frac{4 \cdot n_{\tau}}{\ln \left[n_{\tau}\left[E_{\text {left }}, E_{\text {right }}\right]\right.}\right]$

where $\psi$ is the total number of nearest-neighbor patches, $n_{\tau}$ is the total area of patch $\tau$, and $n_{\tau}\left[E_{\text {left }}, E_{\text {right }}\right]$ are left-diagonal and right-diagonal patch $\tau$ edge pixels.

- $\quad A B R A$ (Average ratio of area to largest bounding rectangle dimension)

$-\quad A B R A=\frac{1}{\psi} \sum_{\tau=1}^{\psi} \frac{\ln \left(n_{\tau}\right)}{Y_{\tau}}$

Here, $\psi$ is the total number of nearest-neighbor patches, $n_{\tau}$ is the total area of patch $\tau$, and the largest and smallest rectangular kernel side length differences $Y_{\tau}$ that would cover a patch $\tau$ is:

$$
Y_{\tau}=\ln \left[\operatorname{MAX}\left(L_{\text {hor }, \tau}, L_{\text {vert }, \tau}\right)\right] \text {. }
$$

34. Average ratio of patch area to area of the circumscribing circle

- $\operatorname{CCRA~(Average~ratio~of~area~to~the~area~of~a~circumscribing~circle~}$

- $\quad C C R A=\frac{1}{\psi} \sum_{\tau=1}^{\psi} \frac{n_{\tau}}{\pi\left(\frac{L_{h o r}, \tau}{2}\right)^{2}}$

where $\psi$ is the total number of nearest-neighbor patches, $n_{\tau}$ is the total area of patch $\tau$, and $L_{h o r, \tau}$ is the longest horizontal diameter of patch $\tau$. 